\title{
Chaos and complexity in quantum mechanics
}

\author{
Tibra Ali, ${ }^{1, *}$ Arpan Bhattacharyya $\odot,{ }^{2,3, \dagger}$ S. Shajidul Haque, ${ }^{4, \risingdotseq}$ Eugene H. Kim, ${ }^{5, \S}$ Nathan Moynihan, ${ }^{4, \|}$ and Jeff Murugan ${ }^{4, \Uparrow}$ \\ ${ }^{1}$ Perimeter Institute, 31 Caroline Street North, Waterloo, Ontario N2L 2Y5, Canada, \\ ${ }^{2}$ Indian Institute of Technology, Gandhinagar, Gujarat 382355, India \\ ${ }^{3}$ Center for Gravitational Physics, Yukawa Institute for Theoretical Physics (YITP), Kyoto University, \\ Kitashirakawa Oiwakecho, Sakyo-ku, Kyoto 606-8502, Japan \\ ${ }^{4}$ The Laboratory for Quantum Gravity \& Strings, Department of Mathematics \& Applied Mathematics, \\ University of Cape Town, Private Bag, Rondebosch 7701, South Africa \\ ${ }^{5}$ Department of Physics, University of Windsor, 401 Sunset Avenue, Windsor, Ontario N9B 3P4 Canada
}

(Received 16 June 2019; published 29 January 2020)

\begin{abstract}
We propose a new diagnostic for quantum chaos. We show that the time evolution of complexity for a particular type of target state can provide equivalent information about the classical Lyapunov exponent and scrambling time as out-of-time-order correlators. Moreover, for systems that can be switched from a regular to unstable (chaotic) regime by a tuning of the coupling constant of the interaction Hamiltonian, we find that the complexity defines a new time scale. We interpret this time scale as recording when the system makes the transition from regular to chaotic behavior.
\end{abstract}

DOI: 10.1103/PhysRevD.101.026021

\section{INTRODUCTION}

Quantum chaos is intrinsically difficult to characterize. Consequently, a precise definition of quantum chaos in many-body systems remains elusive and our understanding of the dynamics of quantum chaotic systems is still inadequate. This lack of understanding is at the heart of a number of open questions in theoretical physics such as thermalization and transport in quantum many-body systems, and black hole information loss. It has also precipitated the renewed interest in quantum chaos from various branches of physics from condensed matter physics to quantum gravity [1].

Chaotic classical systems on the other hand are characterized by their sensitive dependence on initial conditions: two copies of such a system, prepared in nearly identical initial states (namely, two distinct points in phase space, separated by a very small distance), will evolve over time into widely separated configurations. More precisely, the distance between the two points in phase space grows as

\footnotetext{
tali@perimeterinstitute.ca

†abhattacharyya@iitgn.ac.in

${ }^{\ddagger}$ shajid.haque@uwindsor.ca

\$ehkim@uwindsor.ca

"nathanmoynihan@gmail.com

ๆjeff.murugan@uct.ac.za
}

Published by the American Physical Society under the terms of the Creative Commons Attribution 4.0 International license. Further distribution of this work must maintain attribution to the author(s) and the published article's title, journal citation, and DOI. Funded by SCOAP . $\exp \left(\lambda_{L} t\right)$, where $\lambda_{L}$ is the system's largest Lyapunov exponent [2]. This does not happen in quantum mechanics: two nearly identical states, i.e., states with a large initial overlap, remain nearly identical for all time (as their overlap is constant under unitary evolution). It has been argued $[3,4]$ that a quantum analog of "sensitive dependence on initial conditions" is to consider evolving identical states with slightly different Hamiltonians, $\hat{H}$ and $\hat{H}+\delta \hat{H}$. If $\hat{H}$ is the quantization of a (classically) chaotic Hamiltonian, the states will evolve into two different states whose inner product decays exponentially in time.

Traditionally, chaos in quantum systems has been identified by comparison with results from random matrix theory (RMT) [5]. Recently however, other diagnostics have been proposed to probe chaotic quantum systems [6-8]. One such diagnostic is out-of-time-order correlators (OTOCs) $[9,10]$ from which both the (classical) Lyapunov exponent as well as the scrambling time [11-13] may be extracted. However, recent work in mass-deformed Sachdev-Ye-Kitaev (SYK) models [14] have revealed some tension between the OTOC and RMT diagnostics that arise, in part, through the nature of the probes. The OTOC captures early-time quantum mechanical features of the system while RMT diagnostics typically capture late-time statistical features. Evidently, a deeper understanding of probes of quantum chaos is required. In this light, it is interesting therefore to ask whether one can characterize chaos in quantum systems using quantum-informationtheoretic measures. ${ }^{1}$ In this work, we propose a new

\footnotetext{
${ }^{1}$ Some progress in this direction was made in Refs. [15-21].
} 
diagnostic/probe of quantum chaos using the notion of circuit complexity [22-34], adopting Nielsen's geometric approach [35-37]. More specifically, we study the circuit complexity of a particular target state obtained from a reference state by performing a forward evolution followed by a backward evolution with slightly different Hamiltonians. Then we demonstrate how this enables one to probe/characterize chaotic quantum systems, giving information beyond what is contained in the OTOC. Note that instead of using the complexity for a target state that is forward and then backward evolved from a reference state as mentioned above, one can as well study the complexity of a different circuit where both the target and reference states are obtained from time evolution (once) by applying slightly different Hamiltonians from some common state. For circuit complexity from the correlation matrix method that we will be using in this paper, the authors of Ref. [29] concretely showed that the time evolution of complexity in these two scenarios is identical.

To establish out testing method, we consider a simple, exactly solvable system - the inverted oscillator, described by the Hamiltonian $H=p^{2} / 2-\omega^{2} x^{2} / 2$ [38]—which captures the exponential sensitivity to initial conditions exhibited by chaotic systems [39]. Classically, the inverted oscillator has an unstable fixed point at $(x=0, p=0)$; a particle accelerates exponentially away from the fixed point when perturbed. Though the phase-space volume of the inverted oscillator is unbounded, our results are relevant to systems with a bounded phase space in that such a system would be described by an inverted oscillator up to a certain time. The two systems produce the same results over the time of interest (but would not be analytically solvable beyond that time). In what follows, we include the analysis for a regular oscillator as a reference for what arises in a nonchaotic system and explore also a many-body system (quantum field theory) where the inverted oscillator appears. It is worth noting that the inverted quantum oscillator is not just a toy model; it has been realized experimentally [40] and has even played a role in mathematics, in attacking the Riemann hypothesis [41]. It also provides important insights into the bound of the Lyapunov exponents [42].

The rest of the paper is organized as follows. In Sec. II, we present the model and states considered in this work. In Sec. III, we review the ideas behind circuit complexity, and compute the circuit complexity for our system. Section IV demonstrates how quantum chaos can be detected and quantified using circuit complexity while Sec. V discusses the OTOC and its relation to the results obtained from the circuit complexity. In Sec. VI, we discuss a many-body system (quantum field theory) where the inverted oscillator arises. Finally, we summarize and present concluding remarks in Sec. VII.

\section{THE MODEL}

We are interested in comparing the complexity of a regular system with that of an unstable/chaotic system. To that end, we consider the Hamiltonian

$$
H=\frac{1}{2} p^{2}+\frac{\Omega^{2}}{2} x^{2} \quad \text { where } \Omega^{2}=m^{2}-\lambda .
$$

For $\lambda<m^{2}$, Eq. (1) describes a simple harmonic oscillator; for $\lambda>m^{2}$, we have an inverted oscillator. The $\lambda=m^{2}$ case, of course describes a free particle. Our inverted oscillator model can be understood as a short-time approximation for unstable/chaotic systems. In particular, this model captures the exponential sensitivity to initial conditions exhibited by chaotic systems. Let us start with the following state at $t=0$ :

$$
\psi(x, t=0)=\mathcal{N}(t=0) \exp \left(-\frac{\omega_{r} x^{2}}{2}\right),
$$

where

$$
\omega_{r}=m \text {. }
$$

Evolving this state in time by the Hamiltonian (1) produces [43]

$$
\psi(x, t)=\mathcal{N}(t) \exp \left(-\frac{\omega(t) x^{2}}{2}\right),
$$

where $\mathcal{N}(t)$ is the normalization factor and

$$
\omega(t)=\Omega\left(\frac{\Omega-i \omega_{r} \cot (\Omega t)}{\omega_{r}-i \Omega \cot (\Omega t)}\right) .
$$

We will be computing the complexity for this kind of timeevolved state (4) with respect to Eq. (2) and

$$
\omega(t=0)=\omega_{r}
$$

\section{COMPLEXITY FROM THE COVARIANCE MATRIX}

We will start this section with a quick review of circuit complexity and then conclude with a computation of the circuit complexity for a single oscillator. For circuit complexity we will use the covariance matrix method. Note that a similar analysis can be done for circuit complexity from the full wave function.

\section{A. Review of circuit complexity}

Here we will briefly sketch the outline of the computation of circuit complexity. Details of this can be found in Refs. [22,24]. We will highlight only the key formulas and 
interested readers are referred to Refs. [22,24] and citations thereof. The problem is simple enough to state: given a set of elementary gates and a reference state, we want to build the most efficient circuit that starts at the reference state and terminates at a specified target state. Formally,

$$
\left|\psi_{\tau=1}\right\rangle=\tilde{U}(\tau=1)\left|\psi_{\tau=0}\right\rangle,
$$

where

$$
\tilde{U}(\tau)=\overleftarrow{\mathcal{P}} \exp \left(i \int_{0}^{\tau} d \tau H(\tau)\right)
$$

is the unitary operator representing the quantum circuit, which takes the reference state $\left|\psi_{\tau=0}\right\rangle$ to the target state $\left|\psi_{\tau=1}\right\rangle . \tau$ parametrizes a path in the space of the unitaries and given a particular basis (elementary gates) $M_{I}$,

$$
H(\tau)=Y^{I}(\tau) M_{I}
$$

In this context, the coefficients $\left\{Y^{I}(\tau)\right\}$ are referred to as "control functions." The path ordering in Eq. (8) is necessary as all the $M_{I}$ 's do not necessarily commute with each other.

Now, since the states under consideration (2) and (4) are Gaussian, they can be equivalently described by a covariance matrix as follows:

$$
G^{a b}=\left\langle\psi(x, t)\left|\xi^{a} \xi^{b}+\xi^{b} \xi^{a}\right| \psi(x, t)\right\rangle,
$$

where $\xi=\{x, p\}$. This covariance matrix is typically a real symmetric matrix with unit determinant. We will always transform the reference covariance matrix such that $[27,29]$

$$
\tilde{G}^{\tau=0}=S \cdot G^{\tau=0} \cdot S^{T}
$$

where $\tilde{G}^{\tau=0}$ is an identity matrix and $S$ is a real symmetric matrix whose transpose is denoted by $S^{T}$. Similarly, the reference state will transform as

$$
\tilde{G}^{\tau=1}=S \cdot G^{\tau=1} \cdot S^{T} .
$$

The unitary $\tilde{U}(\tau)$ acts on this transformed covariance matrix as,

$$
\tilde{G}^{\tau=1}=\tilde{U}(\tau) \cdot \tilde{G}^{\tau=0} \cdot \tilde{U}^{-1}(\tau)
$$

Next we define a suitable cost function $\mathcal{F}(\tilde{U}, \dot{\tilde{U}})$ and define [22,35-37]

$$
\mathcal{C}(\tilde{U})=\int_{0}^{1} \mathcal{F}(\tilde{U}, \dot{\tilde{U}}) d \tau
$$

Minimizing this cost functional gives us the optimal set of $Y^{I}(\tau)$, which in turn give us the most efficient circuit by minimizing the circuit depth. There are various possible choices for these functions $\mathcal{F}(\tilde{U}, \dot{\tilde{U}})$. For further details, we refer the reader to the extensive literature in Refs. [22-24,35-37]. In this paper, we will choose

$$
\mathcal{F}_{2}(U, Y)=\sqrt{\sum_{I}\left(Y^{I}\right)^{2}}
$$

For this choice, one can easily see that, after minimization the $\mathcal{C}(\tilde{U})$ defined in Eq. (13) corresponds to the geodesic distance on the manifold of unitaries. Note also that we can reproduce our analysis done in the following sections with other choices of the cost functional. We will, however, leave this for future work.

\section{B. Circuit complexity for a single oscillator}

For our case, the covariance matrix corresponding to the target state (4) will take the form,

$$
G^{\tau=1}=\left(\begin{array}{cc}
\frac{1}{\operatorname{Re}(\omega(t))} & -\frac{\operatorname{Im}(\omega(t))}{\operatorname{Re}(\omega(t))} \\
-\frac{\operatorname{Im}(\omega(t))}{\operatorname{Re}(\omega(t))} & \frac{|\omega(t)|^{2}}{\operatorname{Re}(\omega(t))}
\end{array}\right),
$$

where $\omega(t)$ is defined in Eq. (5). For the reference state (2) it will take the following form:

$$
G^{\tau=0}=\left(\begin{array}{cc}
\frac{1}{\omega_{r}} & 0 \\
0 & \omega_{r}
\end{array}\right)
$$

Next we change the basis as follows:

$$
\tilde{G}^{\tau=1}=S \cdot G^{\tau=1} \cdot S^{T}, \quad \tilde{G}^{\tau=0}=S \cdot G^{\tau=0} \cdot S^{T},
$$

with

$$
S=\left(\begin{array}{cc}
\sqrt{\omega^{r}} & 0 \\
0 & \frac{1}{\sqrt{\omega_{r}}}
\end{array}\right),
$$

such that $\tilde{G}^{\tau=0}=I$ is an identity matrix. For the case under study, the reference frequency $\omega_{r}$ is real. We will choose the following three generators:

$M_{11} \rightarrow \frac{i}{2}(x p+p x), \quad M_{22} \rightarrow \frac{i}{2} x^{2}, \quad M_{33} \rightarrow \frac{i}{2} p^{2}$.

These will serve as our elementary gates and satisfy the $S L(2, R)$ algebra,

$$
\begin{aligned}
{\left[M_{11}, M_{22}\right]=} & 2 M_{22}, \quad\left[M_{11}, M_{33}\right]=-2 M_{33}, \\
& {\left[M_{22}, M_{33}\right]=M_{11} . }
\end{aligned}
$$


Next, if we parametrize the $\tilde{U}(\tau)$ as,

$$
\tilde{U}(\tau)=\left(\begin{array}{cc}
\cos (\mu(\tau)) \cosh (\rho(\tau))-\sin (\theta(\tau)) \sinh (\rho(\tau)) & -\sin (\mu(\tau)) \cosh (\rho(\tau))+\cos (\theta(\tau)) \sinh (\rho(\tau)) \\
\sin (\mu(\tau)) \cosh (\rho(\tau))+\cos (\theta(\tau)) \sinh (\rho(\tau)) & \cos (\mu(\tau)) \cosh (\rho(\tau))+\sin (\theta(\tau)) \sinh (\rho(\tau))
\end{array}\right)
$$

and set the boundary conditions as,

$$
\tilde{G}^{\tau=1}=\tilde{U}(\tau=1) \cdot \tilde{G}^{\tau=0} \cdot \tilde{U}^{-1}(\tau=1), \quad \tilde{G}^{\tau=0}=\tilde{U}(\tau=0) \cdot \tilde{G}^{\tau=0} \cdot \tilde{U}^{-1}(\tau=0),
$$

we find that [29],

$$
\{\cosh (2 \rho(1)), \tan (\theta(1)+\mu(1))\}=\left\{\frac{\omega_{r}^{2}+|\omega(t)|^{2}}{2 \omega_{r} \operatorname{Re}(\omega(t))}, \frac{\omega_{r}^{2}-|\omega(t)|^{2}}{2 \omega_{r} \operatorname{Im}(\omega(t))}\right\}, \quad\{\rho(0), \theta(0)+\mu(0)\}=\{0, c\} .
$$

Here $c$ is an arbitrary constant. For simplicity we choose

$$
\mu(\tau=1)=\mu(\tau=0)=0, \quad \theta(\tau=0)=\theta(\tau=1)=c=\tan ^{-1}\left(\frac{\omega_{r}^{2}-|\omega(t)|^{2}}{2 \omega_{r} \operatorname{Im}(\omega(t))}\right) .
$$

From Eq. (8) we have,

$$
Y^{I}=\operatorname{Tr}\left(\partial_{\tau} \tilde{U}(\tau) \cdot \tilde{U}(\tau)^{-1} \cdot\left(M^{I}\right)^{T}\right)
$$

where $\operatorname{Tr}\left(M^{I} \cdot\left(M^{J}\right)^{T}\right)=\delta^{I J}$. Using this we can define the metric

$$
d s^{2}=G_{I J} d Y^{I} d Y^{* J}
$$

where the $G_{I J}=\frac{1}{2} \delta_{I J}$ is known as a penalty factor. Given the form of $U(s)$ in Eq. (21) we will have,

$$
d s^{2}=d \rho^{2}+\cosh (2 \rho) \cosh ^{2} \rho d \mu^{2}+\cosh (2 \rho) \sinh ^{2} \rho d \theta^{2}-\sinh (2 \rho)^{2} d \mu d \theta,
$$

and the complexity functional defined in Eq. (13) will take the form,

$$
\mathcal{C}(\tilde{U})=\int_{0}^{1} d \tau \sqrt{g_{i j} \dot{x}^{i} \dot{x}^{j}}
$$

The simplest solution for the geodesic is again a straight line on this geometry $[22,29]$.

$$
\rho(\tau)=\rho(1) \tau
$$

Evaluating Eq. (28) we simply get

$$
\mathcal{C}(\tilde{U})=\rho(1)=\frac{1}{2}\left(\cosh ^{-1}\left[\frac{\omega_{r}^{2}+|\omega(t)|^{2}}{2 \omega_{r} \operatorname{Re}(\omega(t))}\right]\right) .
$$

This is the geodesic distance in the space of $\operatorname{SL}(2, R)$ unitaries with the end points anchored at the two points determining the boundary conditions (23).

\section{QUANTIFYING CHAOS USING COMPLEXITY}

The goal of this paper is to explore whether we can implement the notion of quantum circuit complexity as a diagnostic of a system's chaotic behavior. Classically, chaos is diagnosed by studying trajectories in the phase space of some dynamical systems, a notion that is not well defined in quantum systems, essentially because of the uncertainty principle. It is important to keep in mind that when we speak of geodesics in the context of circuit complexity, we will mean trajectories defined on the space of unitaries.

Now we propose a new diagnostic for chaotic behavior based on circuit complexity. We consider a target state $\left|\psi_{2}\right\rangle$ obtained by evolving a reference state $\left|\psi_{0}\right\rangle$ forward in time with $\hat{H}$ and then backward in time with $\hat{H}+\delta \hat{H}$,

$$
\left|\psi_{2}\right\rangle=e^{i(\hat{H}+\delta \hat{H}) t} e^{-i \hat{H} t}\left|\psi_{0}\right\rangle .
$$

We would like to compute the complexity $\hat{\mathcal{C}}(\tilde{U})$ of this target state $\left|\psi_{2}\right\rangle$ with respect to the reference state $\left|\psi_{0}\right\rangle$ [29]. For a chaotic quantum system, even if the two Hamiltonians $\hat{H}$ and $\hat{H}+\delta \hat{H}$ are arbitrarily close, $\left|\psi_{2}\right\rangle$ will be quite different from $\left|\psi_{0}\right\rangle$, 


$$
\hat{\mathcal{C}}(\tilde{U})=\frac{1}{2}\left(\cosh ^{-1}\left[\frac{\omega_{r}^{2}+|\hat{\omega}(t)|^{2}}{2 \omega_{r} \operatorname{Re}(\hat{\omega}(t))}\right]\right),
$$

where now

$$
\psi_{2}(x, t)=\hat{\mathcal{N}}(t) \exp \left[-\frac{1}{2} \hat{\omega}(t) x^{2}\right]
$$

and

$\hat{\omega}(t)=i \Omega^{\prime} \cot \left(\Omega^{\prime} t\right)+\frac{\Omega^{\prime 2}}{\sin ^{2}\left(\Omega^{\prime} t\right)\left(\omega(t)+i \Omega^{\prime} \cot \left(\Omega^{\prime} t\right)\right)}$.

In this last expression, $\Omega^{\prime}=\sqrt{m^{2}-\lambda^{\prime}}$ is the frequency associated with the Hamiltonian $H^{\prime}=\frac{1}{2} p^{2}+\frac{\Omega^{\prime 2}}{2} x^{2}$ and $\lambda^{\prime}=\lambda+\delta \lambda$ with $\delta \lambda$ being very small. The time dependence of this complexity demonstrates that there is a clear qualitative difference between a regular oscillator and an inverted oscillator as evident from Figs. 1 and 2. For the regular oscillator we get oscillatory behavior [29-31]; the

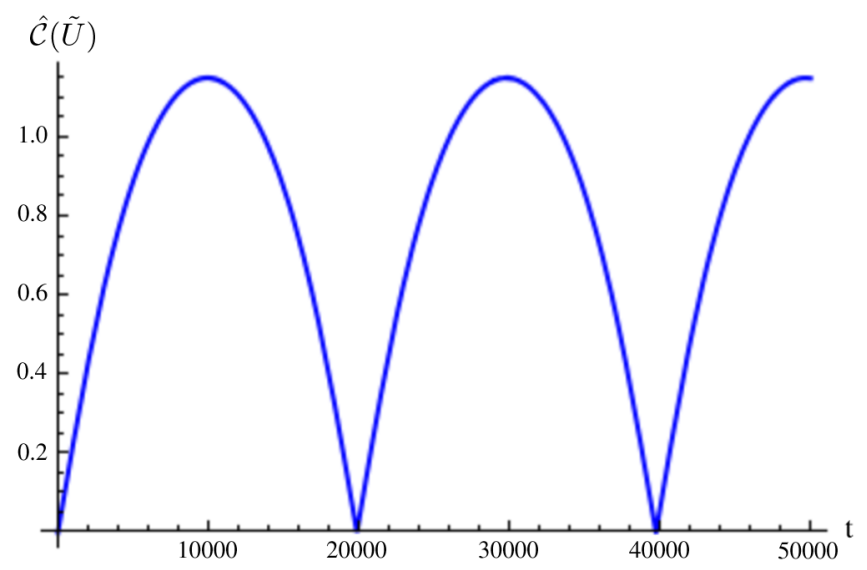

FIG. 1. $\hat{\mathcal{C}}(\tilde{U})$ vs time for regular oscillator $(m=1, \lambda=1.2$, $\delta \lambda=0.01)$.

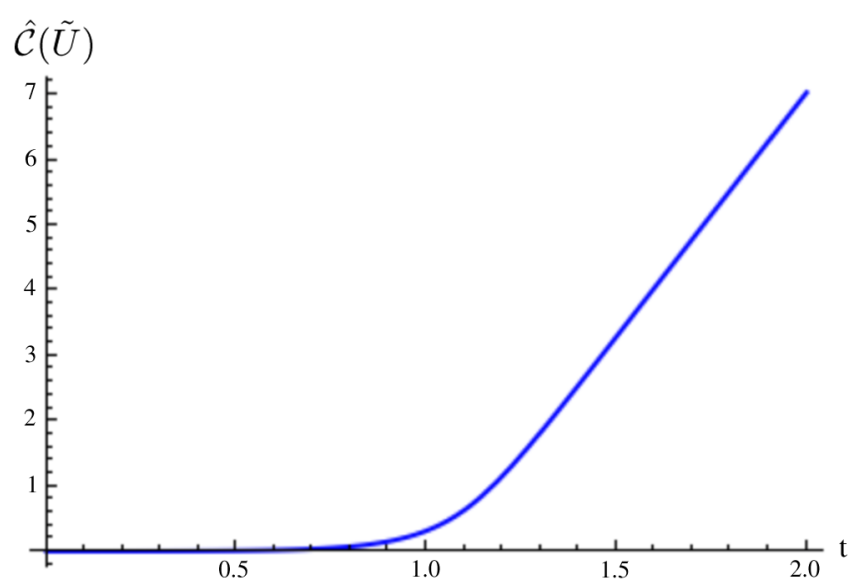

FIG. 2. $\hat{\mathcal{C}}(\tilde{U})$ vs time for inverted oscillator $(m=1, \lambda=15$, $\delta \lambda=0.01)$. complexity grows linearly for a very short period and reaches a saturation with some fluctuations. However, $\hat{\mathcal{C}}(\tilde{U})$ for the inverted oscillator tells a completely different story.

The overall behavior of $\hat{\mathcal{C}}(\tilde{U})$ for the inverted oscillator appears to be some complicated monotonically growing function. However, a closer look at Fig. 2, reveals that it takes a small amount of time for the complexity to pick up

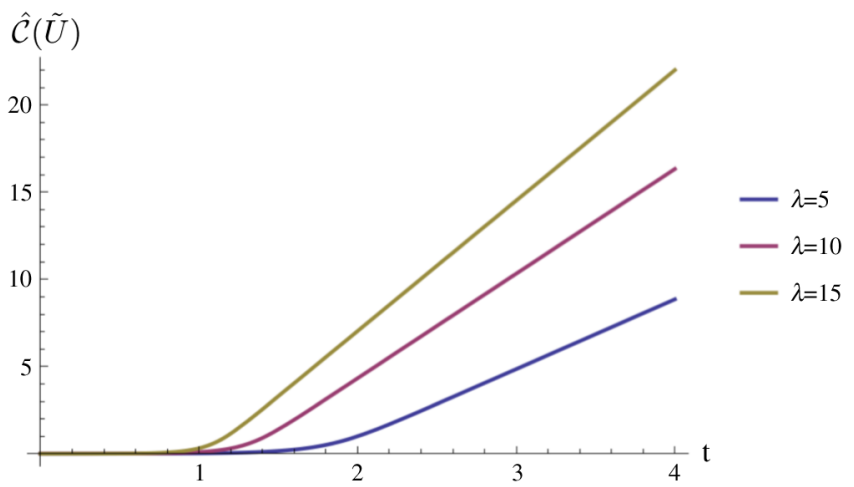

FIG. 3. $\hat{\mathcal{C}}(\tilde{U})$ vs time for different values of $\lambda$ (with $\delta \lambda=0.01$, $m=1)$.

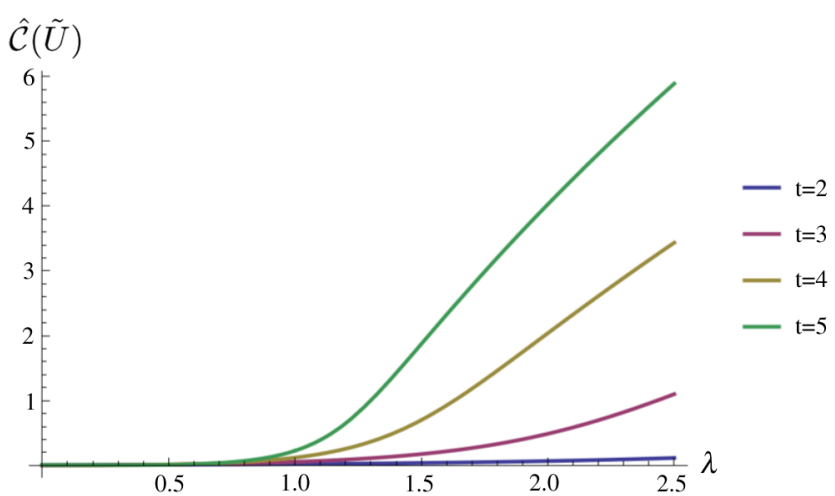

(a)

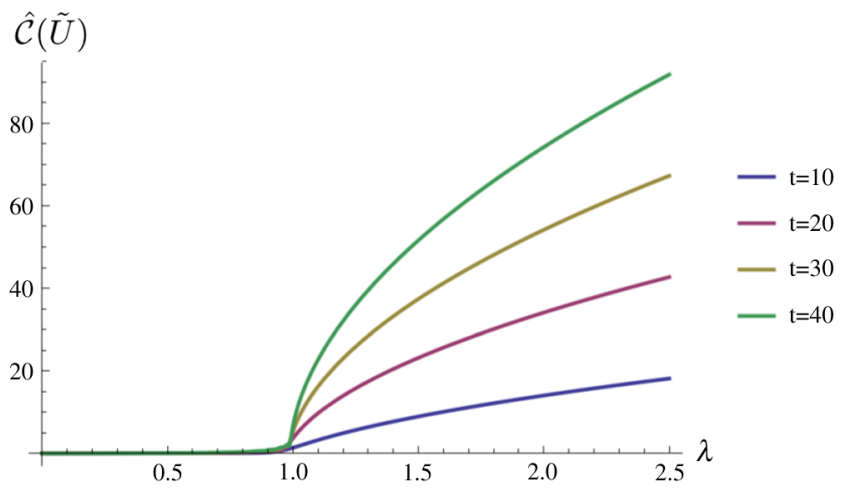

(b)

FIG. 4. Complexity vs $\lambda$ for different times. (a) For all $t, \hat{\mathcal{C}}(\tilde{U})$ starts to increase before the critical of $\lambda$ namely $\lambda=m^{2}$. (b) We can observe that near $t=40$ there is a sharp increase in $\hat{\mathcal{C}}(\tilde{U})$ at the critical value of $\lambda$ ( $\lambda=1$, for the choice of the parameter). We have set $\delta \lambda=0.01$ and $m=1$ in both panels. 
after which it displays a linear ramp with time. For a different choice of coupling $\left(\lambda>\lambda_{c}\right)$ we get similar behavior with different pick-up time and slope $(\phi)$ for the linear ramp. These features are displayed for different values of the coupling in Fig. 3.

As we increase $\lambda$ (beyond the critical value), we are in effect making the model more unstable and consequently from our very specific circuit model we expect a larger complexity and a smaller pick-up time. Therefore, the slope and pick-up time scale are natural candidates for measuring the unstable nature of the inverted oscillator. When we explore the slope $\phi$ of the linear region (as in Fig. 3) for different values of coupling $\lambda$ we find the behavior shown in Fig. 5. In the following section, we will argue that this slope is similar to the Lyapunov exponent.

Note that the linear growth kicks in near a certain time $t=t_{s}$ (as in the Fig. 2) which depends on the choice of the parameters, $\{m, \lambda\}$. We plot this pick-up time as function of $\lambda$ in Fig. 5(b). We believe that this time scale is equivalent to the scrambling time which frequently appears in the chaos literature. One way to confirm this is to compute the outof-time-order four-point correlator. The time when the OTOC $\sim e^{\Lambda\left(t-t_{*}\right)}$ becomes $\mathcal{O}(1)$, is called the scrambling

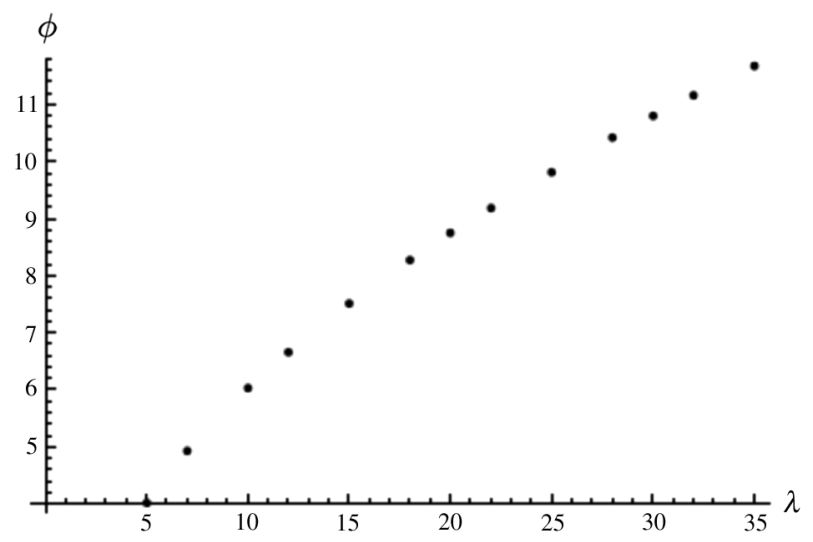

(a)

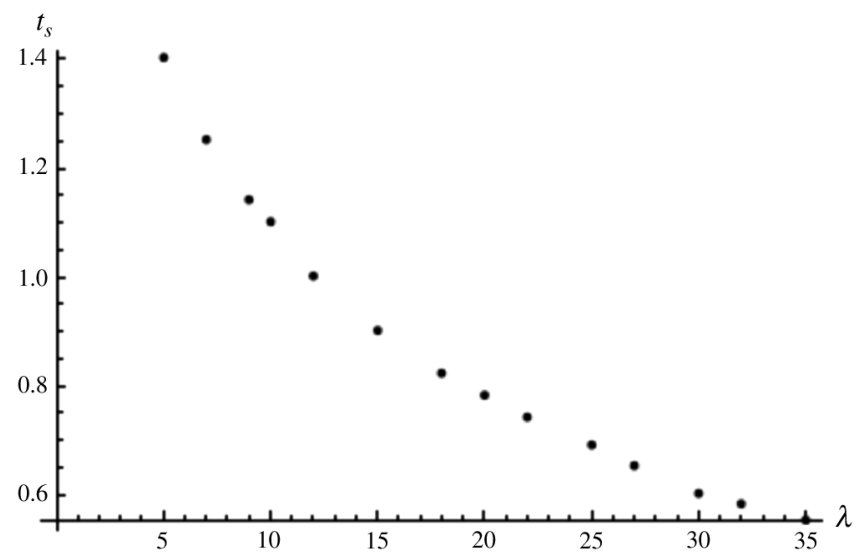

(b)

FIG. 5. (a) Slope $\phi$ vs $\lambda(\delta=0.01, m=1)$. (b) $t_{s}$ vs $\lambda$ $(\delta=0.01, m=1)$.

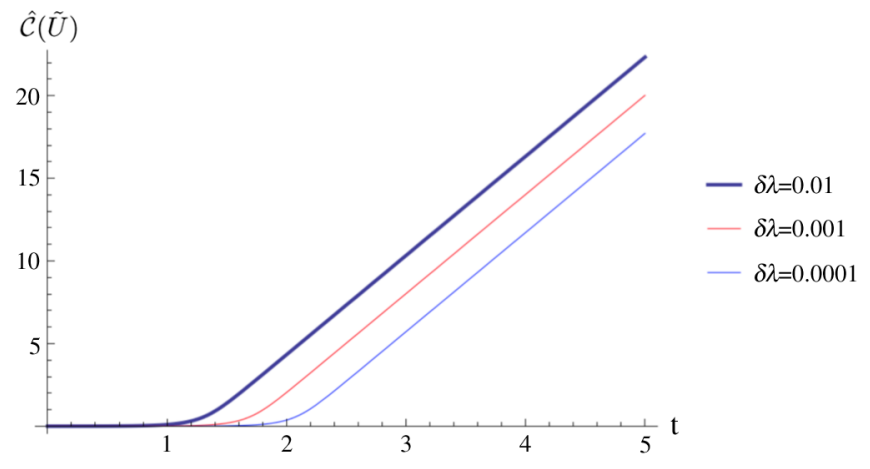

FIG. 6. Dependence of $\hat{\mathcal{C}}(\tilde{U})$ on $\delta \lambda(m=1, \lambda=10)$.

time. For this oscillator model (1), we can show it analytically following Ref. [44]. It is shown in the next section.

Now we will study how this complexity changes with the coupling $\lambda$ for a fixed time. Figure 4 shows how the complexity changes with the coupling $\lambda$ for various times. We denote $\lambda_{c}=m^{2}$ as the critical value of $\lambda$, after which the system becomes an inverted oscillator. We find that for smaller values of $t$ the complexity start to increase for $\lambda<\lambda_{c}$. Around $t=40$ the complexity sharply increases at $\lambda=\lambda_{c}$. We call this time the critical time $t_{c}$. Figure 4 shows that, it takes a certain amount of time for the system to "know" that it has become chaotic; $t_{c}$ marks when this occurs.

We further check the sensitivity of our results to the magnitude of $\delta \lambda$. We plot $\hat{\mathcal{C}}(\tilde{U})$ for the inverted oscillator for a fixed value of $\lambda$ but for different $\delta \lambda$. We find that while the slope of the linear region remains the same, the pick-up time is sensitive to $\delta \lambda$ as exhibited in Fig. 6.

We will conclude this section by highlighting the fact that we get the same result regarding diagnosing chaos when we explore the circuit complexity (using the correlation matrix method), where both the target and reference states are evolved by slightly different Hamiltonians from some other state. The equality between these two complexities was concretely shown in Ref. [29].

\section{OTOC, LYAPUNOV EXPONENT AND SCRAMBLING TIME}

The exponential behavior of the four-point OTOC has recently emerged as a popular early-time diagnostic for quantum chaos. ${ }^{2}$ In Ref. [44] the authors explicitly calculated the OTOCs for a harmonic oscillator. For our model, the OTOC for the $x$ and $p$ operators (after reinstating the factor of $\hbar$ ) gives [44]

\footnotetext{
${ }^{2}$ An alternative to the OTOC, $F(t)=\left\langle A^{\dagger}(t) B^{\dagger}(0) A(t) B(0)\right\rangle$, is the thermally averaged commutator squared $C(t)=$ $\left\langle[A(t), B(0)]^{2}\right\rangle$ with the two being related through $C(t)=2-2 \operatorname{Re}(F(t))$. Unless there is an explicit ambiguity, we will refer to them both as the OTOC.
} 


$$
\left\langle[x(t), p]^{2}\right\rangle=\hbar^{2} \cos ^{2} \Omega t,
$$

where $\Omega$ is defined in Eq. (1). When $\Omega$ is imaginary, we can write the above expression as an exponential function

$$
\left\langle[x(t), p]^{2}\right\rangle \approx \hbar^{2} e^{2|\Omega| t}+\cdots .
$$

Rewriting the above expression as $e^{2 \lambda_{L}\left(t-t_{*}\right)}$, with the Lyapunov exponent $\lambda_{L}$ allows us to immediately read off that for our system, $\lambda_{L}=|\Omega|$ while the scrambling (or Ehrenfest) time is given by

$$
t_{*}=\frac{1}{\lambda_{L}} \log \frac{1}{\hbar} .
$$

The $\lambda$ dependence of this time scale (in units of $\log \frac{1}{\hbar}$ ) is shown in Fig. 7(b). The nature of the graph is in agreement with Fig. 5(b). In fact from Fig. 7(b) after doing a data fitting we get for the pick-up time,

$$
t_{s}=\frac{4 \log (2)}{|\Omega|} .
$$

In the scale of $\log \frac{1}{\hbar}$ this is related to the scrambling time $t_{*}$ as,

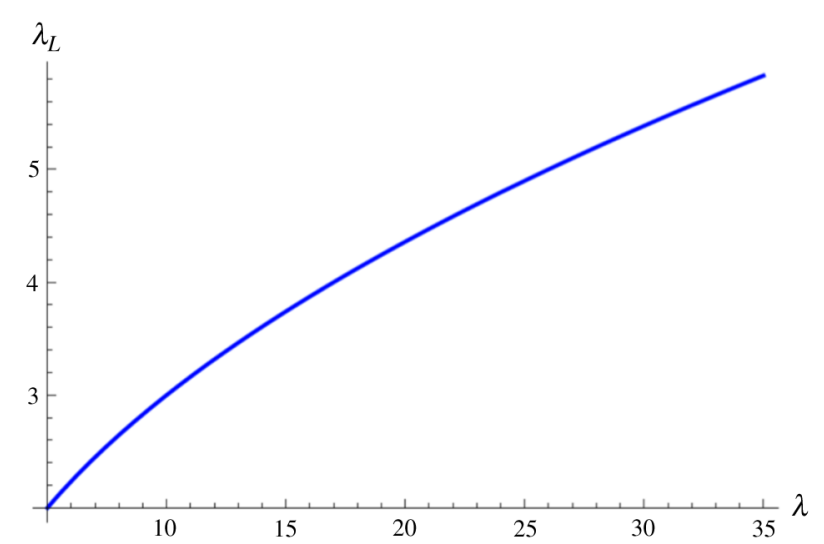

(a)

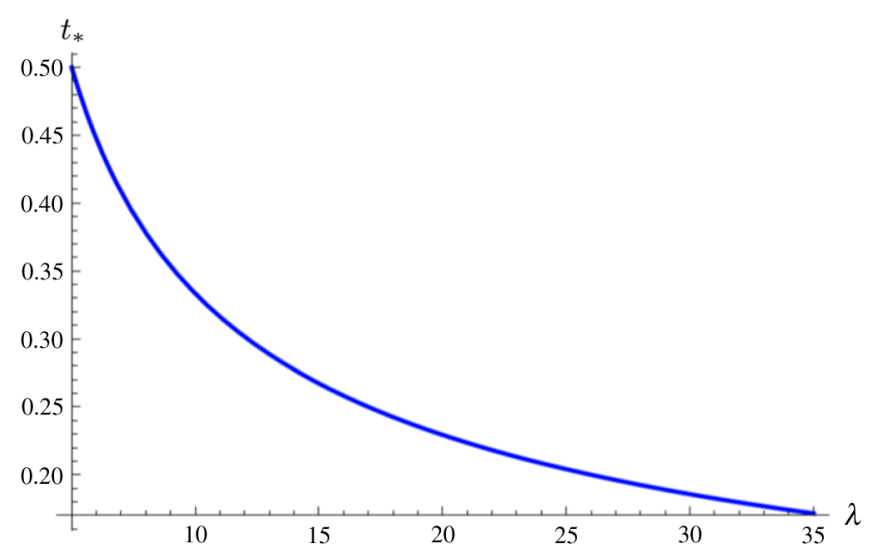

(b)

FIG. 7. (a) $\lambda_{L}$ vs $\lambda(m=1)$. (b) $t_{*}$ vs $\lambda(m=1)$.

$$
t_{s}=4 \log (2) t_{*} .
$$

Also, the $\lambda$ dependence of the Lyapunov exponent is shown in Fig. 7(a). Again the nature of the graph is in agreement with Fig. 5(a). After fitting the data we get for the slope $\phi$ of the linear region of the graph in Fig. 5(a),

$$
\phi=2|\Omega|=2 \lambda_{L} .
$$

We have also checked the $m$ dependence of the slope $\phi$ and the pick-up time $t_{s}$ and they are in agreement with the $m$ dependence of $\lambda_{L}$ and $t_{*}$ respectively.

Before we end this section we would like to highlight the following interesting point. Mathematically, it is evident from Eqs. (35) and (36) that the exponential decay of the OTOC is a consequence of a simple analytic continuation. This is due to the change of $\cos \Omega t$ to $\cosh \Omega t$, when $\Omega$ becomes imaginary. This implies that a simple analytic continuation is essentially capturing the scrambling and chaotic behavior in this quantum system.

\section{TOWARDS A FIELD THEORY ANALYSIS}

By using the single oscillator model we have illustrated how complexity can capture chaotic behavior. In this section we will explore a possible field theory model in which the inverted oscillator appears naturally. Consider two free scalar field theories $[(1+1)$-dimensional $c=1$ conformal field theories] deformed by a marginal coupling. The Hamiltonian is given by

$$
\begin{aligned}
H= & H_{0}+H_{I} \\
= & \frac{1}{2} \int d x\left[\Pi_{1}^{2}+\left(\partial_{x} \phi_{1}\right)^{2}+\Pi_{2}^{2}+\left(\partial_{x} \phi_{2}\right)^{2}+m^{2}\left(\phi_{1}^{2}+\phi_{2}^{2}\right)\right] \\
& +\lambda \int d x\left(\partial_{x} \phi_{1}\right)\left(\partial_{x} \phi_{2}\right) .
\end{aligned}
$$

We can discretize this theory by putting it on a lattice. Then by using the definitions

$$
\begin{aligned}
x(\vec{n}) & =\delta \phi(\vec{n}), \quad p(\vec{n})=\Pi(\vec{n}) / \delta, \quad \omega=m, \\
\Omega & =\frac{1}{\delta^{2}}, \quad \hat{\lambda}=\lambda \delta^{-4}, \quad \hat{m}=\frac{m}{\delta},
\end{aligned}
$$

we get

$$
\begin{aligned}
H= & \frac{\delta}{2} \sum_{n}\left[p_{1, n}^{2}+p_{2, n}^{2}+\left(\Omega^{2}\left(x_{1, n+1}-x_{1, n}\right)^{2}\right.\right. \\
& +\Omega^{2}\left(x_{2, n+1}-x_{2, n}\right)^{2}+\left(\hat{m}^{2}\left(x_{1, n}^{2}+x_{2, n}^{2}\right)\right. \\
& \left.\left.+\hat{\lambda}\left(x_{1, n+1}-x_{1, n}\right)\left(x_{2, n+1}-x_{2, n}\right)\right)\right] .
\end{aligned}
$$

Next we perform a series of transformations, 


$$
\begin{aligned}
& x_{1, a}=\frac{1}{\sqrt{N}} \sum_{k=0}^{N-1} \exp \left(\frac{2 \pi i k}{N} a\right) \tilde{x}_{1, k}, \\
& p_{1, a}=\frac{1}{\sqrt{N}} \sum_{k=0}^{N-1} \exp \left(-\frac{2 \pi i k}{N} a\right) \tilde{p}_{1, k}, \\
& x_{2, a}=\frac{1}{\sqrt{N}} \sum_{k=0}^{N-1} \exp \left(\frac{2 \pi i k}{N} a\right) \tilde{x}_{2, k}, \\
& p_{2, a}=\frac{1}{\sqrt{N}} \sum_{k=0}^{N-1} \exp \left(-\frac{2 \pi i k}{N} a\right) \tilde{p}_{2, k}, \\
& \tilde{p}_{1, k}=\frac{p_{s, k}+p_{a, k}}{\sqrt{2}}, \tilde{p}_{2, k}=\frac{p_{s, k}-p_{a, k}}{\sqrt{2}}, \\
& \tilde{x}_{1, k}=\frac{x_{s, k}+x_{a, k}}{\sqrt{2}}, \tilde{x}_{2, k}=\frac{x_{s, k}-p_{a, k}}{\sqrt{2}},
\end{aligned}
$$

that lead to the Hamiltonian

$$
H=\frac{\delta}{2} \sum_{k=0}^{N-1}\left[p_{s, k}^{2}+\bar{\Omega}_{k}^{2} x_{s, k}^{2}+p_{a, k}^{2}+\Omega_{k}^{2} x_{a, k}^{2}\right],
$$

where

$$
\begin{aligned}
& \bar{\Omega}_{k}^{2}=\left(\hat{m}^{2}+4\left(\Omega^{2}+\hat{\lambda}\right) \sin ^{2}\left(\frac{\pi k}{N}\right)\right), \\
& \Omega_{k}^{2}=\left(\hat{m}^{2}+4\left(\Omega^{2}-\hat{\lambda}\right) \sin ^{2}\left(\frac{\pi k}{N}\right)\right) .
\end{aligned}
$$

It is immediately clear that by tuning the value of $\hat{\lambda}$, the frequencies $\Omega_{k}$ can be made arbitrarily negative resulting in coupled inverted oscillators. Note that $\bar{\Omega}_{k}$ will always be positive. Therefore, one can view Eq. (45) as a sum of regular and inverted oscillators for each value of $k$. With regards to studying the unstable behavior, the regular oscillator part is not very interesting. Hence, we will simply investigate the inverted oscillator part with the Hamiltonian

$$
\tilde{H}(m, \Omega, \hat{\lambda})=\frac{\delta}{2} \sum_{k=0}^{N-1}\left[p_{k}^{2}+\left(\hat{m}^{2}+4\left(\Omega^{2}-\hat{\lambda}\right) \sin ^{2}\left(\frac{\pi k}{N}\right)\right) x_{k}^{2}\right] .
$$

Note that by tuning $\hat{\lambda}$ for this Hamiltonian one can get both regular and inverted oscillators. At $t=0$ we start with the ground state of $\tilde{H}(m, \Omega, \hat{\lambda}=0)$. Then we compute $\hat{\mathcal{C}}(\tilde{U})$ as before by considering two Hamiltonians $\tilde{H}$ and $\tilde{H}^{\prime}$ with two slightly different couplings, $\hat{\lambda}$ and $\hat{\lambda}^{\prime}=\hat{\lambda}+\delta \hat{\lambda}$, where $\delta \hat{\lambda}$ is small. We get

$$
\hat{\mathcal{C}}(\tilde{U})=\frac{1}{2} \sqrt{\sum_{k=0}^{N-1}\left(\cosh ^{-1}\left[\frac{\omega_{r, k}^{2}+\left|\hat{\omega}_{k}(t)\right|^{2}}{2 \omega_{r, k} \operatorname{Re}\left(\hat{\omega}_{k}(t)\right)}\right]\right)^{2}},
$$

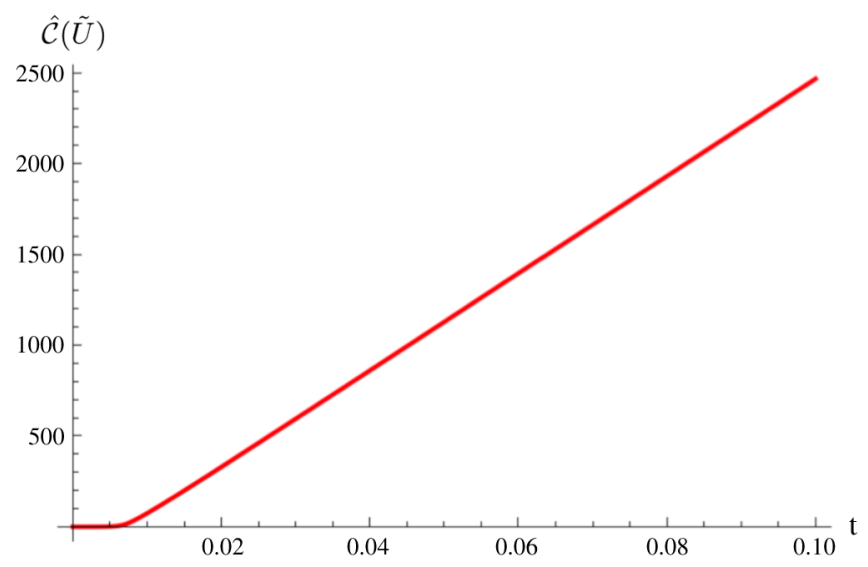

FIG. 8. $\hat{\mathcal{C}}(\tilde{U})$ vs time for the inverted oscillator $(\delta=0.1$, $m=1, N=1000, \lambda=10, \delta \lambda=0.01)$.

where

$$
\begin{aligned}
\hat{\omega}_{k}(t) & =i \Omega_{k}^{\prime} \cot \left(\Omega_{k}^{\prime} t\right)+\frac{\Omega_{k}^{\prime 2}}{\sin ^{2}\left(\Omega_{k}^{\prime} t\right)\left(\omega_{k}(t)+i \Omega_{k}^{\prime} \cot \left(\Omega_{k}^{\prime} t\right)\right)} \\
\Omega_{k}^{\prime 2} & =\hat{m}^{2}+4\left(\Omega^{2}-\hat{\lambda}-\delta \hat{\lambda}\right) \sin ^{2}\left(\frac{\pi k}{N}\right)
\end{aligned}
$$

and $\omega_{k}(t), \omega_{r, k}^{2}$ are defined as,

$$
\omega_{k}(t)=\Omega_{k}\left(\frac{\Omega_{k}-i \omega_{r, k} \cot \left(\Omega_{k} t\right)}{\omega_{r, k}-i \Omega_{k} \cot \left(\Omega_{k} t\right)}\right)
$$

and

$$
\omega_{r, k}^{2}=\hat{m}^{2}+4 \Omega^{2} \sin ^{2}\left(\frac{\pi k}{N}\right)
$$

Using our testing method (outlined in Sec. IV), once again for the inverted oscillator we can immediately read off the scrambling time and Lyapunov exponent from the time evolution of $\hat{\mathcal{C}}(\tilde{U})$ as shown in Fig. 8.

\section{DISCUSSION}

In this paper we used a harmonic oscillator model that converts to an inverted oscillator for large coupling of the interaction Hamiltonian. The coupling behaves as a regulator and by tuning it we can switch between regular and inverted regimes. Our motivation was to use this inverted oscillator as a toy model to study quantum chaos. In this context, the regular oscillator serves as a reference system. We developed a new diagnostic for quantum chaos by constructing a particular quantum circuit and computing the corresponding complexity. Our diagnostic can extract equivalent information as the out-of-time-order correlator with the additional feature that complexity can detect when the system switches from the regular to the chaotic regime. 
We considered a target state which is first forward evolved and then backward evolved with slightly different Hamiltonians and found that the behaviors for the regular and inverted oscillators are completely different in this case. For the regular oscillator we got some oscillatory behavior as in Refs. [28,29,31]. However, for the inverted oscillator we got an exponential-type function with two distinct features: for an initial period the complexity is nearly zero, after which it exhibits a steep linear growth. By comparing with the operator product expansion, we discovered the small time scale and slope of the linear portion to be equivalent to the scrambling time and the Lyapunov exponent respectively. To elaborate further, we note that the only difference between the scrambling time [as in Eq. (37)] and pick-up time [as in Eq. (38)] is just $\mathcal{O}(1)$ [as evident from Eq. (39)] (constant in natural units). Hence, these definitions capture the same scrambling physics.

To give a proof-of-principle argument for complexity as a chaos diagnostic, we have used the inverted oscillator as a toy model. This is, however, a rather special example and, by no means, a realistic chaotic system. To put complexity on the same footing as, say the OTOC as a probe of quantum chaos will take much more work, with more "realistic" systems like the maximally chaotic SYK model $^{3}$ and its many variants (see, for example, Refs. [4648] and references therein) in the $(0+1)$-dimensional quantum mechanical context, or the Murugan-StanfordWitten class of $(1+1)$-dimensional (nonmaximally) chaotic conformal field theories [49].

\footnotetext{
${ }^{3}$ It is worth pointing out that as this manuscript was being prepared, we were made aware of the recent article [45] also advocating for the idea of complexity as a tool for the study of quantum chaotic systems.
}

As a final point of motivation, we note that by virtue of the recent "complexity = action" [50] and "complexity = volume" [51] conjectures, the computational complexity of a holographic quantum system has a well-defined (if not entirely unambiguous) dual. This opens up tantalizing new possibilities in the study of quantum chaos in strongly coupled quantum systems. We leave these issues for future work.

\section{ACKNOWLEDGMENTS}

We would like to thank Dario Rosa and Jon Shock for useful comments. A. B. thanks Aninda Sinha, Pratik Nandy, Jose Juan Fernandez-Melgarejo and Javier Molina Vilaplana for many discussions and ongoing collaborations on complexity. A. B. also thanks the Department of Physics, University of Windsor, Perimeter Institute and the FISPAC group (especially Jose Juan Fernandez-Melgarejo and E. Torrente-Lujan) of the Department of Physics, University of Murcia for their warm hospitality during this work. A. B. is supported by JSPS Grant-in-Aid for JSPS fellows (17F17023). J. M. is supported by the NRF of South Africa under Grant No. CSUR 114599. N. M. is supported by the South African Research Chairs Initiative of the Department of Science and Technology and the National Research Foundation of South Africa. Any opinion, finding and conclusion or recommendation expressed in this material is that of the authors and the NRF does not accept any liability in this regard. Research at Perimeter Institute is supported by the Government of Canada through the Department of Innovation, Science, and Economic Development, and by the Province of Ontario through the Ministry of Research and Innovation.
[1] Interested readers are referred to the following thesis and the references therein; N. Hunter-Jones, Chaos and Randomness in strongly-interacting quantum systems, Ph.D. thesis, California Institute of Technology, 2018, https://thesis .library.caltech.edu/11002/,

[2] F. Haake, Quantum Signatures of Chaos (Springer, New York, 2010).

[3] A. Peres, Quantum Theory: Concepts and Methods, Fundamental Theories of Physics Vol. 72 (Kluwer, Dordrecht, 1995).

[4] R. A. Jalabert and H. M. Pastawski, EnvironmentIndependent Decoherence Rate in Classically Chaotic Systems, Phys. Rev. Lett. 86, 2490 (2001).

[5] O. Bohigas, M. J. Giannoni, and C. Schmit, Characterization of Chaotic Quantum Spectra and Universality of Level Fluctuation Laws, Phys. Rev. Lett. 52, 1 (1984).
[6] S. H. Shenker and D. Stanford, Black holes and the butterfly effect, J. High Energy Phys. 03 (2014) 067.

[7] S. H. Shenker and D. Stanford, Stringy effects in scrambling, J. High Energy Phys. 05 (2015) 132.

[8] A. Kitaev, A simple model of quantum holography, KITP (2015), http://online.kitp.ucsb.edu/online/entangled15/kitaev/, http://online.kitp.ucsb.edu/online/entangled15/kitaev2/.

[9] J. Maldacena, S. H. Shenker, and D. Stanford, A bound on chaos, J. High Energy Phys. 08 (2016) 106.

[10] A. I. Larkin and Y. N. Ovchinnikov, Quasiclassical method in the theory of superconductivity, JETP 28, 1200 (1969), http://www.jetp.ac.ru/cgi-bin/dn/e_028_06_1200.pdf.

[11] P. Hayden and J. Preskill, Black holes as mirrors: Quantum information in random subsystems, J. High Energy Phys. 09 (2007) 120. 
[12] Y. Sekino and L. Susskind, Fast Scramblers, J. High Energy Phys. 10 (2008) 065.

[13] N. Lashkari, D. Stanford, M. Hastings, T. Osborne, and P. Hayden, Towards the fast scrambling conjecture, J. High Energy Phys. 04 (2013) 022.

[14] T. Nosaka, D. Rosa, and J. Yoon, The Thouless time for mass-deformed SYKJ. High Energy Phys. 09 (2018) 041.

[15] M. Miyaji, Butterflies from information metric, J. High Energy Phys. 09 (2016) 002.

[16] P. Hosur, X.-L. Qi, D. A. Roberts, and B. Yoshida, Chaos in quantum channels, J. High Energy Phys. 02 (2016) 004.

[17] D. A. Roberts and B. Yoshida, Chaos and complexity by design, J. High Energy Phys. 04 (2017) 121.

[18] J. Cotler, N. Hunter-Jones, J. Liu, and B. Yoshida, Chaos, complexity, and random matrices, J. High Energy Phys. 11 (2017) 048.

[19] J. M. Magan, Black holes, complexity and quantum chaos, J. High Energy Phys. 09 (2018) 043.

[20] V. Balasubramanian, M. DeCross, A. Kar, and O. Parrikar, Quantum complexity of time evolution with chaotic Hamiltonians, arXiv:1905.05765.

[21] A. Y. Yosifov and L. G. Filipov, Quantum complexity and Chaos in young black holes, Universe 5, 93 (2019).

[22] R. A. Jefferson and R. C. Myers, Circuit complexity in quantum field theory, J. High Energy Phys. 10 (2017) 107.

[23] M. Guo, J. Hernandez, R. C. Myers, and S. M. Ruan, Circuit complexity for coherent states, J. High Energy Phys. 10 (2018) 011.

[24] L. Hackl and R. C. Myers, Circuit complexity for free fermions, J. High Energy Phys. 07 (2018) 139.

[25] R. Khan, C. Krishnan, and S. Sharma, Circuit complexity in Fermionic field theory, Phys. Rev. D 98, 126001 (2018).

[26] S. Chapman, M. P. Heller, H. Marrochio, and F. Pastawski, Toward a Definition of Complexity for Quantum Field Theory States, Phys. Rev. Lett. 120, 121602 (2018).

[27] H. A. Camargo, P. Caputa, D. Das, M. P. Heller, and R. Jefferson, Complexity as a Novel Probe of Quantum Quenches: Universal Scalings and Purifications, Phys. Rev. Lett. 122, 081601 (2019).

[28] A. Bhattacharyya, A. Shekar, and A. Sinha, Circuit complexity in interacting QFTs and RG flows, J. High Energy Phys. 10 (2018) 140.

[29] T. Ali, A. Bhattacharyya, S. Shajidul Haque, E. H. Kim, and N. Moynihan, Time evolution of complexity: A critique of three methods, J. High Energy Phys. 04 (2019) 087.

[30] T. Ali, A. Bhattacharyya, S. Shajidul Haque, E. H. Kim, and N. Moynihan, Post-quench evolution of distance and uncertainty in a topological system: Complexity, entanglement and revivals, arXiv:1811.05985.

[31] S. Chapman, J. Eisert, L. Hackl, M. P. Heller, R. Jefferson, H. Marrochio, and R. C. Myers, Complexity and entanglement for thermofield double states, SciPost Phys. 6, 034 (2019).

[32] K. Hashimoto, N. Iizuka, and S. Sugishita, Time evolution of complexity in Abelian gauge theories-and playing quantum Othello game, Phys. Rev. D 96, 126001 (2017); R.-Q. Yang, A complexity for quantum field theory states and application in thermofield double states, Phys. Rev. D 97, 066004 (2018); R.-Q. Yang, C. Niu, C.-Y. Zhang, and K.-Y. Kim, Comparison of holographic and field theoretic complexities for time dependent thermofield double states,
J. High Energy Phys. 02 (2018) 082; D. W. F. Alves and G. Camilo, Evolution of complexity following a quantum quench in free field theory, arXiv:1804.00107; J. Jiang, J. Shan, and J. Yang, Circuit complexity for free Fermion with a mass quench, arXiv:1810.00537; R. Q. Yang and K. Y. Kim, Complexity of operators generated by quantum mechanical Hamiltonians, J. High Energy Phys. 03 (2019) 010; S. Liu, Complexity and scaling in quantum quench in $1+1$ dimensional fermionic field theories, arXiv:1902.02945.

[33] P. Caputa, N. Kundu, M. Miyaji, T. Takayanagi, and K. Watanabe, Anti-de Sitter Space from Optimization of Path Integrals in Conformal Field Theories, Phys. Rev. Lett. 119, 071602 (2017); Liouville action as path-integral complexity: From continuous tensor networks to AdS/CFT, J. High Energy Phys. 11 (2017) 097; B. Czech, Einstein Equations from Varying Complexity, Phys. Rev. Lett. 120, 031601 (2018); J. Molina-Vilaplana and A. Del Campo, Complexity functionals and complexity growth limits in continuous MERA circuits, arXiv:1803.02356; A. Bhattacharyya, P. Caputa, S. R. Das, N. Kundu, M. Miyaji, and T. Takayanagi, Path-integral complexity for perturbed CFTs, J. High Energy Phys. 07 (2018) 086; T. Takayanagi, Holographic spacetimes as quantum circuits of path-integrations, J. High Energy Phys. 12 (2018) 048; H. A. Camargo, M. P. Heller, R. Jefferson, and J. Knaute, Path Integral Optimization as Circuit Complexity, Phys. Rev. Lett. 123, 011601 (2019).

[34] R. Q. Yang, Y. S. An, C. Niu, C. Y. Zhang, and K. Y. Kim, Axiomatic complexity in quantum field theory and its applications, arXiv:1803.01797; P. Caputa and J. M. Magan, Quantum computation as gravity, arXiv:1807.04422; R. Q. Yang, Y. S. An, C. Niu, C. Y. Zhang, and K. Y. Kim, More on complexity of operators in quantum field theory, J. High Energy Phys. 03 (2019) 161; A. Bernamonti, F. Galli, J. Hernandez, R. C. Myers, S. M. Ruan, and J. Simn, The first law of complexity, arXiv:1903.04511; I. Akal, Weighting gates in circuit complexity and holography, arXiv:1903.06156; G. Camilo, D. Melnikov, F. Novaes, and A. Prudenziati, Circuit complexity of Knot states in Chern-Simons theory, arXiv:1903.10609; A. R. Brown and L. Susskind, The complexity geometry of a single qubit, arXiv:1903.12621; D. Ge and G. Policastro, Circuit complexity and 2D Bosonisation, arXiv:1904.03003; W. H. Huang, Operator approach to complexity: Excited state, arXiv:1905.02041; V. Balasubramanian, M. DeCross, A. Kar, and O. Parrikar, Binding complexity and multiparty entanglement, J. High Energy Phys. 02 (2019) 069.

[35] M. A. Nielsen, A geometric approach to quantum circuit lower bounds, arXiv:quant-ph/0502070.

[36] M. A. Nielsen, M. R. Dowling, M. Gu, and A. M. Doherty, Quantum computation as geometry, Science 311, 1133 (2006).

[37] M. A. Nielsen and M. R. Dowling, The geometry of quantum computation, arXiv:quant-ph/0701004.

[38] G. Barton, Quantum mechanics of the inverted oscillator, Ann. Phys. (N.Y.) 166, 322 (1986).

[39] R. Blume-Kohout and W. Zurek, Decoherence from a chaotic environment: An upside-down oscillator as a model, Phys. Rev. A 68, 032104 (2003).

[40] S. Gentilini, M. C. Braidotti, G. Marcucci, E. DelRe, and C. Conti, Physical realization of the Glauber quantum oscillator, Sci. Rep. 5, 15816 (2015). 
[41] M. V. Berry and J.P. Keating, The Riemann zeros and eigenvalue asymptotics, SIAM Rev. 41, 236 (1999).

[42] T. Morita, Thermal Emission from Semi-classical Dynamical Systems, Phys. Rev. Lett. 122, 101603 (2019); Semiclassical bound on Lyapunov exponent and acoustic Hawking radiation in $c=1$ matrix model, arXiv:1801.00967.

[43] See and e. g. R. Shankar, Principles of Quantum Mechanics (Plenum, New York, 1994).

[44] K. Hashimoto, K. Murata, and R. Yoshii, Out-of-time-order correlators in quantum mechanics, J. High Energy Phys. 10 (2017) 138.

[45] V. Balasubramanian, M. DeCross, A. Kar, and O. Parrikar, Quantum complexity of time evolution with Chaotic Hamiltonians, arXiv:1905.05765.

[46] J. Maldacena and D. Stanford, Remarks on the SachdevYe-Kitaev model, Phys. Rev. D 94, 106002 (2016).
[47] W. Fu, D. Gaiotto, J. Maldacena, and S. Sachdev, Supersymmetric Sachdev-Ye-Kitaev models, Phys. Rev. D 95, 026009 (2017); Erratum, 95, 069904 (2017).

[48] A. Kitaev and S. J. Suh, The soft mode in the Sachdev-YeKitaev model and its gravity dual, J. High Energy Phys. 05 (2018) 183.

[49] J. Murugan, D. Stanford, and E. Witten, More on Supersymmetric and $2 \mathrm{~d}$ analogs of the SYK model, J. High Energy Phys. 08 (2017) 146.

[50] A. R. Brown, D. A. Roberts, L. Susskind, B. Swingle, and Y. Zhao, Holographic Complexity Equals Bulk Action? Phys. Rev. Lett. 116, 191301 (2016).

[51] D. A. Roberts, D. Stanford, and L. Susskind, Localized shocks, J. High Energy Phys. 03 (2015) 051. 